\title{
Characteristics of intramural metastasis in gastric cancer
}

\author{
Tatsuya Hashimoto $\cdot$ Kuniyoshi Arai • \\ Yuichi Yamashita $\cdot$ Yoshiaki Iwasaki • \\ Tsunekazu Hishima
}

Received: 16 May 2012/Accepted: 17 December 2012/Published online: 13 January 2013

(C) The International Gastric Cancer Association and The Japanese Gastric Cancer Association 2013

\begin{abstract}
Background Intramural metastasis (IM) in gastric cancer is rare. However, it often occurs with esophageal squamous cell carcinoma and has been reported to have a poor prognosis.

Methods In 4,714 cases of gastric cancer that underwent gastrectomy, the clinicopathological features and postoperative prognoses of 29 cases with IM were evaluated and compared with 2,770 cases of advanced gastric cancer without IM.

Results Of the 4,714 cases, $29(0.6 \%)$ were histopathologically diagnosed with gastric cancer with IM. There were significant differences in the number of lymph node
\end{abstract}

T. Hashimoto $(\bowtie) \cdot$ Y. Yamashita

Department of Gastroenterological Surgery, Fukuoka University

School of Medicine, 7-45-1 Nanakuma, Jonan-ku,

Fukuoka 814-0180, Japan

e-mail: adams7230@me.com

K. Arai

Tokyo Metropolitan Health and Medical Treatment Corporation

Toshima Hospital, 33-1 Sakae-cho, Itabashi-ku,

Tokyo 173-0015, Japan

Y. Iwasaki

Department of Surgery, Tokyo Metropolitan Cancer and Infectious Diseases Center, Komagome Hospital, 3-18-22

Honkomagome, Bunkyo-ku, Tokyo 113-8677, Japan

T. Hishima

Department of Pathology, Tokyo Metropolitan Cancer and Infectious Diseases Center, Komagome Hospital, 3-18-22

Honkomagome, Bunkyo-ku, Tokyo 113-8677, Japan metastases, capillary invasion, and stage grouping between cases with IM and advanced gastric cancer without IM. Metastasis size was approximately within $2 \mathrm{~cm}$, and many metastases occurred within $2 \mathrm{~cm}$ of the primary lesion. Multiple metastases were observed in $38 \%$ of cases and occurred mainly in the submucosa and muscularis propria. IM was detected preoperatively in $\mathbf{1 7 . 2} \%$ of cases and was present equally on both sides of the primary lesion. Nine cases had IM outside the stomach. The median survival time with IM was significantly less than in cases of advanced gastric cancer without IM $(p<0.0001)$. A subgroup of cases with IM within $1 \mathrm{~cm}$ of the primary lesion had a relatively favorable prognosis.

Conclusions The presence of IM is thought to be one of the most important prognostic factors in gastric cancer. Aggressive resection is recommended to increase longterm survival if curative resection is possible.

Keywords Stomach neoplasms - Gastric cancer . Lymphatic metastasis - Intramural metastasis · Prognosis

\section{Introduction}

Intramural metastasis (IM) in gastric cancer has been rarely reported. However, esophageal squamous cell carcinoma is often accompanied by IM, and such cases have been reported to have a poor prognosis. As a result, the presence of IM with carcinoma is considered to be one of the most important prognostic factors [1-3]. There have been no detailed clinicopathological descriptions of IM in gastric cancer, to date. Therefore, we retrospectively conducted a clinicopathological study on the incidence of IM and its relationship to prognosis and survival. 


\section{Patients and methods}

We reviewed 29 cases with IM in gastric cancer among 4,714 cases $(0.6 \%)$ who underwent gastrectomy between 1975 and 2004 at the Tokyo Metropolitan Cancer and Infectious Diseases Center (CICK), Komagome Hospital. Clinicopathological characteristics including age, gender, operation data, histological diagnosis, stage, and survival data were obtained from our hospital database and clinical records. Preoperative diagnosis was based on upper gastrointestinal barium studies, endoscopic examination, and computed tomography. Resected specimens were examined using standard hematoxylin and eosin staining. The gastric cancers were evaluated according to the "Japanese Classification of Gastric Carcinoma" (Japanese Gastric Cancer Association, 3rd English edition) [4].

\section{Diagnostic criteria of IM}

To diagnose IM histologically, we modified the criteria of Nishimaki et al. [1] as follows: (1) clearly separated from the primary tumor; (2) located in the wall of the esophagus, stomach, or duodenum; (3) having a gross appearance of a submucosal tumor without intraepithelial cancer extension; (4) having the same histological type as the primary tumor; and (5) lacking any evidence of intravascular growth.

These criteria discriminated IMs from multiple primary tumors in the esophagus or stomach and from intravascular tumor emboli around the primary tumor. A typical example of IM is shown in Fig. 1.

\section{Statistical analysis}

The association between factors was evaluated using the $\chi^{2}$ test and Fisher's exact probability test. The significance of difference among means was determined using the Mann-Whitney $U$ test. Survival rates were determined using the Kaplan-Meier method, and differences between survival curves were examined with the log-rank test. Statistical analyses were performed using the SAS software package Stat View, version 5.0 (SAS Institute, Cary, NC, USA). A result was considered statistically significant when the $p$ value was $<0.05$.

\section{Results}

From 1975 to $2004,4,714$ patients with gastric cancers underwent gastrectomy at the CICK. Surgeries were as follows: 1,691 cases of total gastrectomy; 2,721 cases of
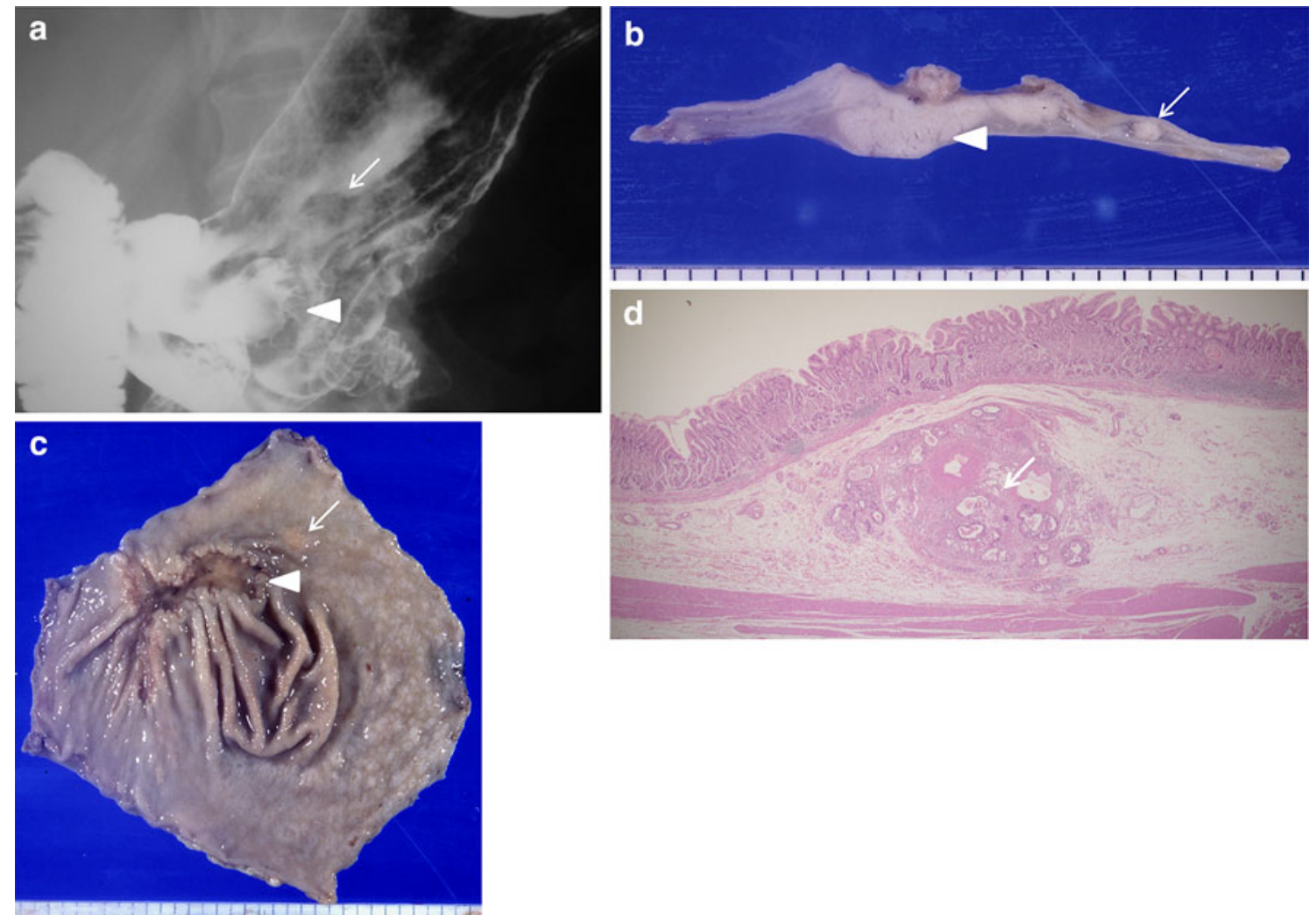

Fig. 1 A case of gastric cancer with intramural metastasis (IM). X-ray examination (a) and gross appearance of the resected specimen (b, c) show the primary tumor (arrowheads) and IM (arrows). The IM is clearly separated from the primary tumor and has the gross appearance of a submucosal tumor. d Histologically, IM (arrow) is present in the submucosal layer. Hematoxylin and eosin (H\&E) 
distal gastrectomy; 162 cases of proximal gastrectomy; 94 cases of segmentectomy; 11 cases of pancreaticoduodenectomy; 32 cases of pylorus-preserving gastrectomy; and 3 cases of segmental resection of the stomach. There were 1,915 cases of early cancer and 2,799 cases of advanced cancer. All 29 cases of IM were seen in advanced cancers, providing an onset frequency of $0.6 \%$ of all resection cases and $1 \%$ of advanced cancer cases. In IM cases, surgeries were as follows: 17 cases of total gastrectomy, 11 cases of distal gastrectomy, and 1 case of proximal gastrectomy.

The clinicopathological characteristics of gastric cancer with IM

To evaluate the clinicopathological characteristics of gastric cancer with IM, 29 cases of gastric cancer with IM were compared with 2,770 cases of advanced gastric cancer without IM (Table 1). The mean age of IM cases is higher than cases of advanced gastric cancer without IM. Histologically, IM cases occurred significantly more frequently in number of lymph node metastases, lymphatic invasion, vessel invasion, and advanced stage groupings compared with cases of advanced gastric cancer without IM. All IM cases showed marked lymph node metastasis and capillary invasion. There were no significant differences between these two groups with respect to gender, the main location of the primary tumor, tumor size, macroscopic type, histological type, depth of invasion, and residual tumor.

\section{Characteristics of IM}

The characteristics of IM are shown in Table 2. The mean IM size was $1.09 \pm 1.10 \mathrm{~cm}$ (range, $0.2-6.0 \mathrm{~cm}$ ). The number of IMs was one in 18 cases $(62 \%)$, two in 4 cases, three in 4 cases, four in 1 case, and five or more in 2 cases (multiple, $38 \%$ ). The layers affected by IM were 13 cases

Table 1 Patient characteristics of gastric cancer cases

\begin{tabular}{|c|c|c|c|c|c|}
\hline & \multicolumn{2}{|l|}{ With IM $(n=29)$} & \multicolumn{2}{|c|}{ Advanced gastric cancer without IM } & \multirow[t]{2}{*}{$p$ value } \\
\hline & $N$ & Percent $(\%)$ & $N$ & Percent $(\%)$ & \\
\hline Gender (male/female) & $20 / 9$ & $68 / 32$ & $1,816 / 954$ & $66 / 34$ & 0.70 \\
\hline \multicolumn{6}{|l|}{ Age } \\
\hline Mean \pm SD (range) & $66.4 \pm 9.12(48-81)$ & - & $60.7 \pm 12.26(24-92)$ & & 0.04 \\
\hline \multicolumn{6}{|l|}{ Main location of primary tumor } \\
\hline Upper/middle/lower & $10 / 10 / 9$ & $34 / 34 / 32$ & $791 / 1,018 / 961$ & $29 / 37 / 34$ & 0.78 \\
\hline Tumor size $(\mathrm{cm})$ & $\begin{array}{r}8.22 \pm 4.02 \\
(2.3-18.0)\end{array}$ & - & $8.12 \pm 4.19(1.2-25.0)$ & & 0.89 \\
\hline \multicolumn{6}{|l|}{ Macroscopic type } \\
\hline $1 / 2 / 3 / 4 / 5$ & $2 / 5 / 15 / 4 / 3$ & 7/17/52/14/10 & 97/760/988/433/492 & $4 / 27 / 35 / 16 / 18$ & 0.82 \\
\hline \multicolumn{6}{|l|}{ Histological type } \\
\hline Pap/tub1/tub2/por/sig/muc & $4 / 3 / 9 / 9 / 2 / 2$ & $14 / 10 / 31 / 31 / 7 / 7$ & 321/429/507/988/366/159 & 12/15/18/36/13/6 & 0.51 \\
\hline \multicolumn{6}{|l|}{ Depth of invasion } \\
\hline $\mathrm{m} / \mathrm{sm} / \mathrm{mp} / \mathrm{ss} / \mathrm{se} / \mathrm{si}$ & $0 / 0 / 2 / 5 / 15 / 7$ & $0 / 0 / 7 / 17 / 52 / 24$ & 0/0/285/1,031/1,076/378 & $0 / 0 / 10 / 37 / 39 / 14$ & 0.07 \\
\hline \multicolumn{6}{|l|}{ Number of lymph node metastases } \\
\hline $0 / 1-2 / 3-6 / 7 \leq$ & $0 / 2 / 8 / 19$ & $0 / 7 / 28 / 65$ & $604 / 541 / 592 / 1,033$ & $22 / 20 / 21 / 37$ & 0.0016 \\
\hline $\begin{array}{l}\text { Distant metastasis site (liver/peritoneal/ } \\
\text { CY1) }{ }^{\mathrm{a}}\end{array}$ & $11(5 / 8 / 1)$ & - & $(168 / 477 /-)$ & - & NA \\
\hline \multicolumn{6}{|l|}{ Capillary invasion } \\
\hline ly0/ly $1 / 1 y 2 / l y 3$ & $0 / 2 / 7 / 20$ & $0 / 7 / 24 / 69$ & $73 / 1,137 / 870 / 690$ & $3 / 41 / 31 / 25$ & $<0.0001$ \\
\hline $\mathrm{v} 0 / \mathrm{v} 1 / \mathrm{v} 2 / \mathrm{v} 3$ & $0 / 4 / 17 / 8$ & $0 / 14 / 59 / 27$ & 273/1,338/840/317 & $10 / 48 / 30 / 22$ & $<0.0001$ \\
\hline \multicolumn{6}{|l|}{ Residual tumor } \\
\hline $\mathrm{R} 0 / \mathrm{R} 1, \mathrm{R} 2$ & $14 / 15$ & $48 / 52$ & $1,753 / 1,017$ & $63 / 37$ & 0.12 \\
\hline \multicolumn{6}{|l|}{ Stage } \\
\hline I B/II A/II B/III A/III B/IIIC/IV & $0 / 1 / 2 / 3 / 3 / 11 / 11$ & $\begin{array}{l}0 / 4 / 0 / 10 / 10 / 38 / \\
38\end{array}$ & $\begin{array}{l}184 / 331 / 352 / 346 / 358 / 283 / \\
916\end{array}$ & $\begin{array}{l}7 / 12 / 13 / 12 / 13 / 10 / \\
33\end{array}$ & 0.0001 \\
\hline
\end{tabular}

$I M$ intramural metastasis, pap papillary adenocarcinoma, tub 1 well-differentiated tubular adenocarcinoma, tub 2 moderately differentiated tubular adenocarcinoma, por poorly differentiated adenocarcinoma, sig signet-ring cell carcinoma, $m u c$ mucinous cell carcinoma, $m$ mucosa, $s m$ submucosa, $m p$ muscularis propria, ss subserosa, se serosa exposed, se adjacent structures, $N A$ not assessable

${ }^{\text {a }}$ With overlapping 
(45\%) in the submucosa (sm), 10 cases (34\%) in the muscularis propria (mp), 5 cases $(17 \%)$ in the subserosa (ss), and 1 (4\%) case in the serosa exposed (se). IM was detected preoperatively in 5 cases $(17.2 \%)$ using upper gastrointestinal barium studies and endoscopic examination. The mean distance between the IM and the primary tumor was $1.21 \pm 0.94 \mathrm{~cm}$ (range, $0.1-5.5 \mathrm{~cm}$ ). Eleven cases had IM in the portion of the surgical specimen proximal to the primary tumor, 11 cases had IM in the distal portion, and 7 cases had IM on both sides. Nine cases had IM in an adjacent organ, 6 cases had IM in the esophageal wall, and 3 cases had IM in the duodenal wall.

Prognosis of the IM cases

The median survival time for all gastric cancers with IM was 11 months (with a $13.9 \%$ survival rate at 3 years). Survival time was 13.5 months (with an $18.8 \%$ survival rate at 3 years) for cases in which $\mathrm{R} 0$ resection was obtained, and 7.5 months (with an $8.9 \%$ survival rate at 3 years) for cases in which $\mathrm{R} 1 / 2$ resection was obtained, with no significant

Table 2 Characteristics of intramural metastasis (IM)

\begin{tabular}{ll}
\hline Size $(\mathrm{cm})$ & \\
Mean \pm SD (range) & $\begin{array}{c}1.09 \pm 1.10 \\
(0.2-6.0)\end{array}$ \\
Number of IM & $18 / 4 / 4 / 1 / 2$ \\
$1 / 2 / 3 / 4 / \geq 5$ & \\
Affected layer of IM & $13 / 10 / 5 / 1$ \\
$\quad$ sm/mp/ss/se & \\
Distance from primary tumor $(\mathrm{cm})$ & $1.21 \pm 0.94(1-5.5)$ \\
$\quad$ Mean \pm SD (range) & $5(17.2)$ \\
Preoperative diagnosis $(\%)$ & \\
Direction from primary tumor & $11 / 11 / 7$ \\
$\quad$ Anal/oral/both sides & $3 / 6$ \\
Located in an adjacent organ esophagus/ \\
duodenum
\end{tabular}

difference observed between the two groups $(p=0.12)$ (Fig. 2). However, no cases of recurrence were observed in 5 of 14 cases in which $\mathrm{R} 0$ resection was obtained.The median survival time for all gastric cancers with IM was significantly lower than for cases of advanced gastric cancer without IM $(p<0.0001)$. In cases of advanced gastric cancer without IM, the median survival time was 39.4 months with a $51.1 \%$ survival rate at 3 years (Fig. 3).

Prognosis by distance from the primary lesion and by the size of the IM was also calculated. The relationship between the size of the IM and the distance from the primary lesion is shown in Fig. 4. A constant tendency was not observed between the IM size and the distance from the primary lesion, but the IM size was approximately within $2 \mathrm{~cm}$, and many occurred within $2 \mathrm{~cm}$ of the primary lesion. Thus, when the cutoff value for the distance was set at various lengths from 0.5 to $2 \mathrm{~cm}$, the $1-\mathrm{cm}$ distance was associated with the most significant difference in survival. Cases with a distance from the primary tumor of less than $1 \mathrm{~cm}$ lived significantly longer than cases with a distance of $1 \mathrm{~cm}$ or more from the primary tumor. In all cases of IM for the distance from the primary tumor, 3-year survival rates and median survival times were $25.7 \%$ and 28 months versus $0 \%$ and 8 months $(p=0.0026)$, respectively. In $\mathrm{R} 0$ cases of $\mathrm{IM}$ for the distance from the primary tumor, 3-year survival rates and median survival times were $41.7 \%$ and 29 months versus $0 \%$ and 11 months, respectively ( $p=0.0075$ ) (Fig. 5).

Three-year survival rates and median survival times of cases with an IM size smaller than $1 \mathrm{~cm}$ were higher than those of cases with IMs of $1 \mathrm{~cm}$ or larger, although the difference was not significant. In all cases of IM for the IM size, 3-year survival rates and median survival times were $22.1 \%$ and 13 months versus $0 \%$ and 9 months, respectively ( $p=0.36$ ). In R0 cases of IM for IM size, 3-year survival rates and median survival times were $20.0 \%$ and 14.5 months versus not assessable and 11.5 months, respectively ( $p=0.72$ ) (Fig. 6).
Fig. 2 Postoperative survival of IM cases. 3 yr 3-year survival rate, MST mean survival time

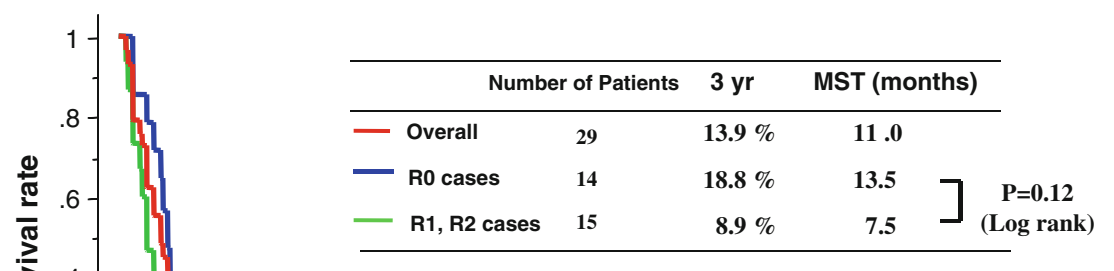




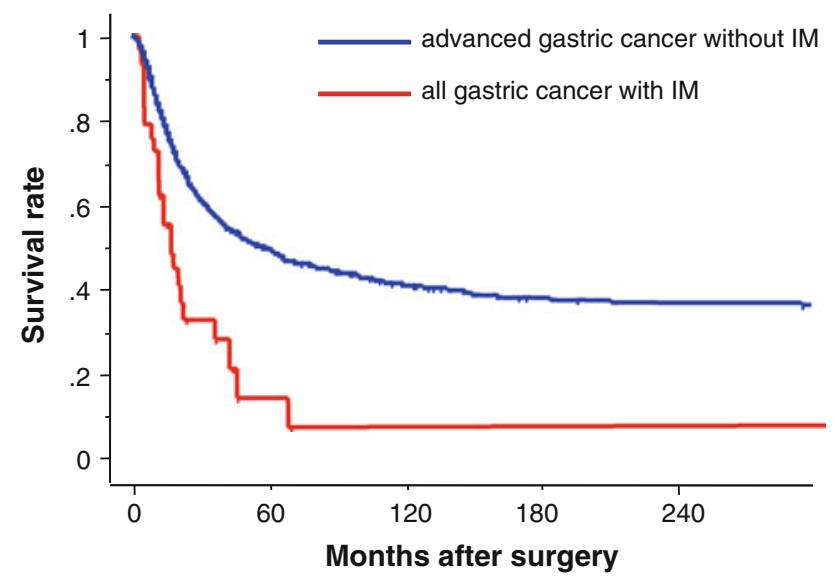

Fig. 3 Survival curves for patients with all gastric cancers with IM (blue line, $n=29$ ) and advanced gastric cancer without IM (red line, $n=2,770): p<0.0001$

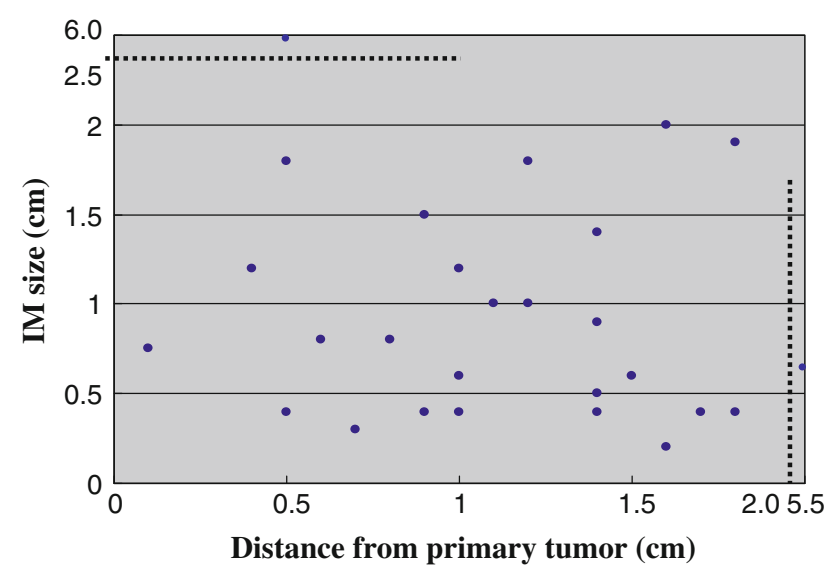

Fig. 4 Correlation between size of IM and distance from primary tumor. The majority of IMs were less than $2 \mathrm{~cm}$ in size and were located within $2 \mathrm{~cm}$ of the primary tumor

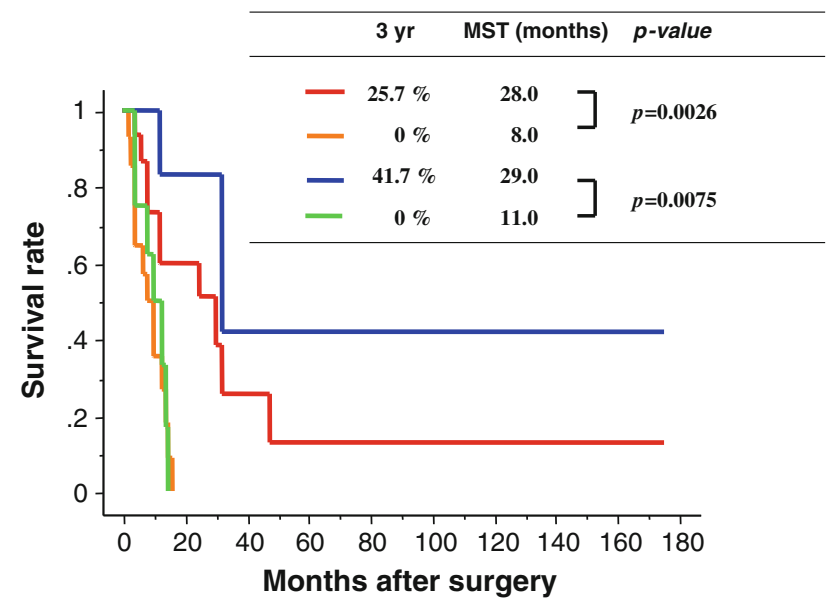

Fig. 5 Postoperative survival according to distance from primary tumor. Red line (15 patients): $<1 \mathrm{~cm}$ from the primary tumor of all cases, orange line (14 patients): $\geq 1 \mathrm{~cm}$ from the primary tumor of all cases, blue line ( 6 patients): $<1 \mathrm{~cm}$ from the primary tumor of $\mathrm{R} 0$ cases, green line ( 8 patients): $\geq 1 \mathrm{~cm}$ from the primary tumor of $\mathrm{R} 0$ cases

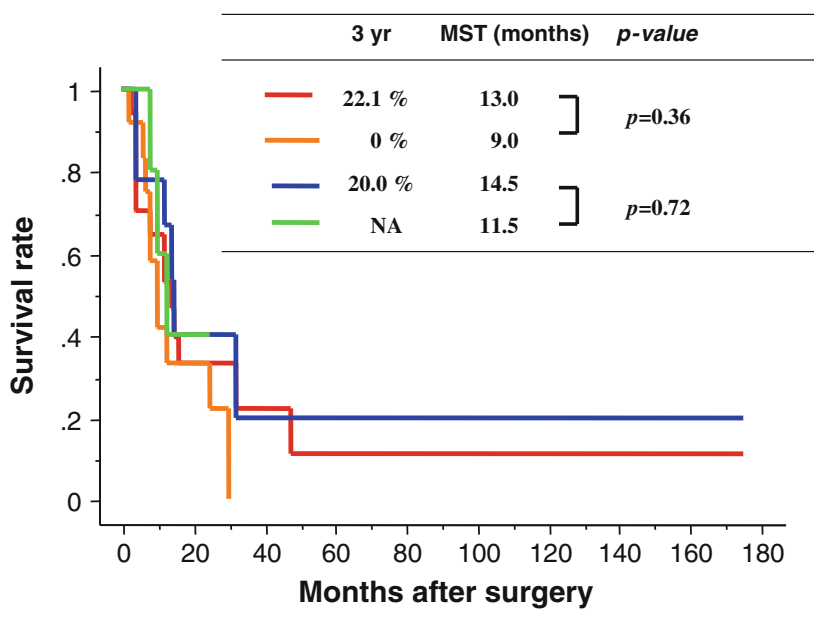

Fig. 6 Postoperative survival according to size of IM. Red line (17 patients): $<1 \mathrm{~cm}$ in size (all cases), orange line (12 patients): $\geq 1 \mathrm{~cm}$ in size (all cases), blue line ( 6 patients): $<1 \mathrm{~cm}$ in size of $\mathrm{R} 0$ cases, green line ( 8 patients): $\geq 1 \mathrm{~cm}$ in size of R0 cases. $N A$ not assessable

\section{Discussion}

Generally, IM in gastric adenocarcinoma is very rare. A PubMed search from 1950 to 2010 using gastric cancer and IM as keywords revealed only three case reports [5, 6], and there are no articles to date that have verified this condition. This study provided the first analysis of IM in gastric cancer.

In contrast to IM in gastric cancer, IM is often observed in esophageal squamous cell carcinoma and was first reported by Watson in 1933 [7]. The frequency reportedly ranges from 10.8 to $15.5 \%$ in advanced esophageal cancers $[2,3,8,9]$. In this study, the incidence of IM was only $0.6 \%$ in cases of resected gastric cancer and $1 \%$ in advanced cancer cases, and it was a very rare pathology, compared with the incidence in esophageal cancer.

Compared with primary lesions without IM in esophageal squamous cell carcinoma, the clinicopathological features of primary lesions with IM are reported to include advanced stage grouping, a higher rate of lymph node metastasis (79-99\%), and a higher rate of capillary invasion $(76-100 \%)[1-3,9]$, features that correspond to the results of this study in gastric cancer. Distant metastasis was detected in $37.9 \%$ of IM in this study, whereas the reported rate of IM is $29.2 \%$ in esophageal cancer [2].

It is believed that IM in esophageal cancer is mediated by lymphatic invasion to the submucosal layer. Consequently, it is believed that lymphatic invasion and/or lymph node metastasis cases are common, with large numbers of lymph node metastases [7]. In this study, based on the fact that cases positive for capillary invasion are common, particularly for lymphatic invasion, the proliferation of cancer cells deeper than the submucosal layer via lymph 
flow was also assumed to be an onset mechanism for IM in gastric cancer, similar to esophageal cancer.

In this study, the IM size was approximately within $2 \mathrm{~cm}$, and many occurred within $2 \mathrm{~cm}$ of the primary lesion. Multiple IMs were observed in $38 \%$ of IM cases, and $31.0 \%$ of IM cases had IM outside the stomach. Some literature states that gastrointestinal endoscopy should be used first, because it is convenient and allows for histological examinations [10]. Especially for advanced gastric cancers, careful observation within $2 \mathrm{~cm}$ of the primary lesion and observation of adjacent organs for the presence of IM will be important for determining the line of dissection of the stomach. However, caution is required because there are cases in which IM is observed inside a wall that is relatively far from the primary lesion, at a maximum of $5.5 \mathrm{~cm}$, or in which metastasis to the duodenum or the esophagus is observed.

Regarding the positional relationship between the primary lesion and the IM in esophageal cancers, Takubo et al. [2] reported that IM was equally present on both sides of the primary lesion. Similarly, in our study, IM was present equally on both sides.

In this study, the IM was detected preoperatively in five cases $(17.2 \%)$. All IMs existed in layers deeper than the sm, so submucosal tumors covered with normal epithelium are an important finding in gastrointestinal barium studies and endoscopic examinations. Takubo et al. [2] reported that the IM in esophageal cancer was detected preoperatively $54.2 \%$ of the time, with a dome-like appearance covered with normal epithelium, similar to that of submucosal tumors, often with erosion or ulceration. The difficulties in making a preoperative diagnosis of IM in gastric cancer probably result from the small size and the absence of erosion and ulceration.

The prognosis of esophageal cancer accompanied by IM is exceedingly poor, with a survival rate at 5 years of $9 \%$ and a median survival time of 7 months [3], making it one of the major causes of poor postoperative prognosis [1, 2]. The median survival time of gastric cancer cases with IM was relatively favorable, at 11 months $(13.9 \%$ survival rate at 3 years). However, the results of our study indicated a significantly poorer prognosis in all gastric cancers with IM than in cases of advanced gastric cancer without IM. A subgroup of cases with IM within $1 \mathrm{~cm}$ of the primary lesion had a relatively favorable prognosis in this study. Yuasa et al. [3] also reported that a subgroup of cases with IM less than $2.0 \mathrm{~cm}$ from the primary tumor may have a relatively favorable prognosis in esophageal cancer. More specifically, even if a gastric cancer is accompanied by IM, long-term survival may be achieved if curability can be obtained and if the IM remains in the vicinity of the primary lesion.

This study has several limitations. Because of the low overall number of cases diagnosed with IM in gastric cancer, the number of cases in our study was too small to perform more rigorous statistical evaluation, and bias may have affected the clinicopathological investigation. However, the method of histopathological evaluation was consistent, and this consistency can be considered a strong point of the study.

In conclusion, the presence of IM is thought to be one of the most important pathways of tumor spread and a factor in determining the prognosis of gastric cancer cases. In this study, $1 \%$ of advanced gastric cancer cases had IM. If the IM is diagnosed preoperatively and curative resection is possible, aggressive resection should be performed. If the IM is not diagnosed preoperatively, surgical margins of at least $2 \mathrm{~cm}$ or more should be obtained.

Acknowledgments We greatly appreciate Dr. Hiroshi Kawachi for providing interpretations of the histopathological findings. We also appreciate Dr. So Katayanagi and Dr. Seiichiro Hoshino for their writing assistance.

Conflict of interest None.

\section{References}

1. Nishimaki T, Suzuki T, Tanaka Y, Aizawa K, Hatakeyama K, Muto T. Intramural metastases from thoracic esophageal cancer: local indicators of advanced disease. World J Surg. 1996;20: $32-7$.

2. Takubo K, Sasajima K, Yamashita K, Tanaka Y, Fujita K. Prognostic significance of intramural metastasis in patients with esophageal carcinoma. Cancer (Phila). 1990;65:1816-9.

3. Yuasa N, Miyake H, Yamada T, Oda K, Nimura Y, Nagasaka T, et al. Prognostic significance of the location of intramural metastasis in patients with esophageal squamous cell carcinoma. Langenbecks Arch Surg. 2004;389:122-7.

4. Japanese Gastric Cancer Association. Japanese classification of gastric carcinoma: 3rd English edition. Gastric Cancer 2011;14:101-112.

5. Ikeda O, Toh Y, Aoki Y, Harimoto N, Taomoto J, Masuda T, et al. Multiple and metachronous esophageal intramural metastases from a gastric adenocarcinoma. Gastric Cancer. 2008;11: 119-22.

6. Szanto I, Voros A, Nagy P, Gonda G, Gamal EM, Altorjay A, et al. Esophageal intramural metastasis from adenocarcinoma of the gastroesophageal junction. Endoscopy. 2002;34:418-20.

7. Watson WL. Carcinoma of the esophagus. Surg Gynecol Obstet. 1933;56:884-97.

8. Kato H, Tachimori $\mathrm{Y}$, Watanabe H, Itabashi M, Hirota $\mathrm{T}$, Yamaguchi $\mathrm{H}$, et al. Intramural metastasis of thoracic esophageal carcinoma. Int J Cancer. 1992;50:49-52.

9. Kuwano H, Watanabe M, Sadanaga N, Kamakura T, Nozoe T, Yasuda M, et al. Univariate and multivariate analyses of the prognostic significance of discontinuous intramural metastasis in patients with esophageal cancer. J Surg Oncol. 1994;57:17-21.

10. Hirota T, Nishimaki T, Suzuki T, Komukai S, Kuwabara S, Aizawa K, et al. Esophageal intramural metastasis from an adenocarcinoma of the gastric cardia: report of a case. Surg Today. 1998;28:1160-2. 